\title{
Presencia y Resistencia a los Antimicrobianos de serovariedades de Salmonella enterica aisladas en una empresa avícola integrada del Ecuador
}

\author{
Sandra Villagómez Estrada ${ }^{1}$, María Logacho Pilataxi ${ }^{1}$, Christian Vinueza Burgos ${ }^{1 *}$ \\ ${ }^{1}$ Facultad de Medicina Veterinaria y Zootecnia, Universidad Central del Ecuador. Quito-Ecuador. \\ *cvinueza@uce.edu.ec
}

doi: $10.26807 /$ remcb.v38i1.17

Recibido 01-07-2016 ; Aceptado 20-01-2017

\begin{abstract}
RESUMEN.- A nivel mundial Salmonella es la responsable de causar millones de casos de gastroenteritis humana y más de cien mil defunciones cada año. Una de las principales fuentes de contagio esta bacteria son los productos de origen aviar. La utilización generalizada de antimicrobianos en la industria avícola ha favorecido el surgimiento de cepas de Salmonella multirresistentes. Dichas cepas podrían transmitirse al ser humano a través del consumo de productos cárnicos contaminados. El objetivo de esta investigación fue identificar serotipos y patrones de resistencia antimicrobiana de cepas de Salmonella aisladas en una empresa avícola integrada en Ecuador. Al final del período de muestreo se recolectaron 289 muestras que fueron analizadas en base a la norma NTE INEN-ISO 6579 Anexo D. El $20.1 \%$ de las muestras fueron positivas a Salmonella, identificándose 4 diferentes serovariedades, siendo $S$. Infantis $(74.1 \%)$ el serotipo más frecuente. La mayoría de cepas de Salmonella presentaron resistencia a nitrofurantoína $(94.8 \%)$, tetraciclina $(82.8 \%)$, cloranfenicol (79.3\%) y trimetopin-sulfametoxazol (81\%). Los resultados de este estudio indican que Salmonella puede estar presente en los diferentes procesos de la industria avícola ecuatoriana, además la resistencia antibacteriana de cepas aisladas en carne de pollo puede representar un riesgo para la salud pública.
\end{abstract}

PALABRAS CLAVES: Ecuador, Industria Avícola, Resistencia a los antimicrobianos, Salmonella, Serotipo.

ABSTRACT.- Salmonella is the cause of millions of cases of human gastroenteritis worldwide and over one hundred thousand deaths each year. A major source of this bacteria are poultry products. The widespread use of antibiotics in poultry industry has favored the emergence of multidrug-resistant strains of Salmonella. Such strains could be transmitted to humans through consumption of contaminated meat products. The objective of this research was to identify serotypes and antimicrobial resistance patterns of Salmonella strains isolated from an integrated poultry company in Ecuador. A total of 289 samples were analyzed with the ISO 6579 Annex D protocol. The results showed $20.1 \%$ of positive samples. Four serotypes were found from which $S$. Infantis $(86.2 \%)$ was the most frequent serotype. Most Salmonella strains were resistant to nitrofurantoin (94.8 \%), tetracycline (82.8\%), chloramphenicol (79.3\%) and trimetopin-sulfamethoxazole $(81 \%)$. The results shown in this study indicate that Salmonella may be present in different processes of Ecuador's poultry industry and that antibacterial resistance strains isolated from chicken meat may represent a risk to public health.

KEYWORDS: Antimicrobial Resistance, Ecuador, Poultry Industry, Salmonella, Serotype. 


\section{INTRODUCCIÓN}

Las bacterias del género Salmonella tienen como hábitat el intestino de seres humanos y animales, pero también pueden encontrarse en el ambiente y en cualquier material con contaminación fecal (OIE 2012, Da Silva et al. 2013).

Salmonella es uno los principales agentes causales de enfermedades diarreicas. Se estima que este patógeno causa más de 78 millones de casos de salmonelosis no tifoidea y 59000 muertes (WHO 2015). La fuente más común de esta bacteria es la carne de pollo, seguida por la carne de cerdo y bovino (EFSA y ECDC 2015b).

El surgimiento de bacterias farmacorresitentes, entre ellas Salmonella, se ha visto favorecido por la utilización generalizada de agentes antibacterianos en animales de abasto, convirtiéndose en un problema para la salud pública a nivel mundial (Su et al. 2004, Rahmani et al. 2013).

En la producción avícola los antibióticos son empleados para la prevención y tratamiento de enfermedades o como promotores de crecimiento, lo que podría favorecer la selección y difusión de bacterias resistentes a los mismos (Gyles 2008, OMS 2014). Las aves de corral pueden albergar cepas resistentes de Salmonella, dichas cepas podrían transmitirse al ser humano a través del consumo de productos avícolas contaminados (Al-Zenki et al. 2007).

Así, se han encontrado cepas resistentes de Salmonella en diferentes etapas de la cadena productiva avícola. Dichas cepas han sido aisladas de muestras de granja, contenido cecal, carcasas y carne de pollo (Mainali et al. 2014, Tîrziu et al. 2015, Voss-Rech et al. 2015, Cui et al. 2016).

Diferentes estudios demuestran que cepas de Salmonella aisladas en la industria avícola presentan resistencia a por lo menos a un agente antibacteriano. En la Unión Europea (UE) se han aislado cepas de Salmonella resistentes al ácido nalidíxico, sulfonamidas y tetraciclinas (EFSA y ECDC 2015a).

En Brasil, cepas asiladas de granjas de pollos presentaron multirresistencia a diferentes antibióticos, entre ellos trimetoprin/sulfametoxazol
(Voss-Rech et al. 2015), mientras que en Colombia más del $96 \%$ de los aislamientos de Salmonella recuperados de granjas (hisopados de barrido y heces) presentaron resistencia a tetraciclinas (Donado-Godoy et al. 2012).

Adicionalmente, en muestras de carcasas depollo, carne,y contenido cecal se han encontrado cepas de Salmonella resistentes principalmente a ácido nalidíxico y tetraciclinas (Sodagari, et al. 2015, Lee et al. 2016, Vinueza et al. 2016)

La industria avícola es uno de los sectores pecuarios de más rápido crecimiento en todo el mundo. El consumo de productos cárnicos avícolas es cada vez mayor (Herren 2012). En el Ecuador se estima que el consumo per cápita de carne de pollo es de $35 \mathrm{~kg} / \mathrm{año}$ (CONAVE 2013).

A pesar de la importancia de este patógeno, son escasas las investigaciones acerca la presencia y resistencia a los antibióticos de Salmonella en los sistemas de producción avícola en Ecuador. El objetivo del presente estudio fue investigar la presencia, serotipos y resistencia antibacteriana de Salmonella aislada a partir muestras de alimento balanceado, materias primas, muestras de granja y carcasas de pollo en una empresa avícola integrada en Ecuador.

\section{METODOLOGÍA}

En el presente estudio, el número de muestras y sitios de muestreos fueron consensuados con la empresa avícola integrada para que representen una cantidad proporcional del número de procesos en la cría de pollos de engorde. Se tomaron muestras en tres etapas de producción en la empresa avícola. La primera etapa consta de muestras tomadas en la planta productora de balanceado. $\mathrm{La}$ segunda etapa constituye las granjas de crianza, tomando muestras en recepción y en finalización. La tercera etapa comprende las muestras tomadas de las carcasas de las aves durante su sacrificio en la planta de faenamiento (Tabla 1). Las muestras fueron procesadas en el Laboratorio de Bacteriología de la Facultad de Medicina Veterinaria y Zootecnia de la Universidad Central del Ecuador.

Toma de Muestras.- Las muestras de alimento terminado, materias primas ensacadas (harina de car- 
ne, harina de sangre, harina triple y pasta de soya) y maíz, fueron colectadas desde la tolva durante la descarga de un lote de alimento en 12 tomas, introduciendo un calador estéril en los sacos y tomando muestras en el tornillo sin fin, respectivamente.

Con la finalidad de evaluar la contaminación inicial de los pollitos de un día de edad se tomaron diez papeles de recibimiento por cada lote de reproductoras. En el laboratorio se colectó aproximadamente $25 \mathrm{~g}$ de papel con contenido fecal para su aislamiento bacteriológico. En la etapa de crianza entre 28-30 días, se evaluó la contaminación de las aves tomando 2 muestras de cada galpón mediante hisopos de barrido. En la planta de faenamiento se tomaron 25 ciegos al azar de cada lote examinado. Posteriormente, en el laboratorio se colectó asépticamente un gramo de heces por cada ciego hasta formar un pool de $25 \mathrm{~g}$.

En la planta de faenamiento se colectaron asépticamente 4 muestras de piel de la pechuga de carcasas antes de entrar al tanque de pre enfriamiento y 4 muestras a su salida del tanque de enfriamiento. Cada muestra estuvo compuesta de un pool de tres porciones de piel provenientes de carcasas diferentes. El peso aproximado de cada muestra fue de $25 \mathrm{~g}$.

Aislamiento e Identificación de Salmonella.- El procesamiento de las muestras en el laboratorio se realizó en base a la norma NTE INEN-ISO 6579 anexo D "Microbiología de los alimentos para consumo humano y alimentación animal. Método horizontal para la detección de Salmonella spp."

Para cada muestra se realizó una dilución de 1:10 con Agua Peptonada Tamponada (BPW; Difco, BD, Sparks, MD) y se homogenizó. Después de la incubación a $37^{\circ} \mathrm{C}$ por 20 horas, se colocó 3 gotas del cultivo enriquecido en una placa de agar semisólido Modified Rappaport-Vassiliadis (MSRV; Oxoid, Basingstoke, UK) y se incubó a $42^{\circ} \mathrm{C}$ por 24 horas. Las placas de agar fueron examinadas para observar la formación de un halo blanquecino. Se tomó una azada desde el borde del halo, se estrió en un placa de agar Xilosa Lisina Desoxicolato (XLD, Difco, $\mathrm{BD}$, Sparks, MD) y se incubó a $37^{\circ} \mathrm{C}$ por 24 horas. Dos colonias presuntivas de Salmonella fueron confirmadas mediante su reacción en agar Hierro Triple Azúcar (TSI, Difco, BD), agar Hierro Lisina (LIA,

Tabla 1. Tipos de muestras analizadas

\begin{tabular}{llll}
\hline Sitio de muestreo & Tipo de muestra & $\begin{array}{l}\text { Número } \\
\text { muestreos }\end{array}$ & $\begin{array}{l}\text { Total } \\
\text { muestras }\end{array}$ \\
\hline Planta de Alimento & Alimento terminado & 22 & 40 \\
& Harina de carne & 13 & 18 \\
& Harina de sangre & 13 & 20 \\
& Harina triple* & 19 & 67 \\
& Maíz & 22 & 30 \\
& Pasta de Soya & 14 & 19 \\
Crianza en granja & Recepción & 29 & 29 \\
& Hisopos de barrido & 6 & 12 \\
& Heces & 6 & 6 \\
Planta de Faenamiento & Piel antes de pre & 6 & 24 \\
& enfriamiento & 6 & 24 \\
\hline Total & Piel después de & 156 & 289 \\
\hline
\end{tabular}

*Harina Triple: mezcla de harina de vísceras, plumas y sangre. 
BBL, BD), Agar Urea (BBL, BD) y medio para reacción de Indol (BBL, BD). Una colonia positiva a la identificación fenotípica de Salmonella se utilizó en los siguientes análisis.

Tipificación serológica de Salmonella.- La tipificación se realizó mediante el esquema de Kauffmann-White que divide a este género bacteriano en diferentes serotipos en base a los antígenos somáticos $(\mathrm{O})$, flagelares $(\mathrm{H})$ y capsular (Vi) (Becton Dickinson, New Jersey- USA.

Sensibilidad a los Antimicrobianos.- La susceptibilidad antimicrobiana se evaluó mediante el método de difusión en disco con 13 agentes antimicrobianos (Oxoid, UK), siguiendo las directrices del Instituto de Normas Clínicas y de Laboratorio (CLSI 2015). Los antimicrobianos utilizados fueron: gentamicina, estreptomicina, penicilina, ampicilina, amoxicilina-ácido clavulánico, cefalotina, cefotaxima, cloranfenicol, azitromicina, ciprofloxacina trimetoprim-sulfametoxazol, tetraciclina y nitrofurantoína. Se utilizó como control la cepa de Escherichia coli ATCC 25922. Se usó los cut offs recomendados por el Instituto de Normas Clínicas y de Laboratorio (CLSI 2015) para determinar la resistencia de las cepas de Salmonella a los antibióticos probados. (Tabla 2). Para identificar los patrones de resistencia se tomó en cuenta a las cepas con fenotipos resistentes e intermedios.

\section{RESULTADOS}

Las muestras de piel tomadas antes del tanque de pre enfriamiento y después del tanque de enfriamiento presentaron el mayor porcentaje de aislamiento a Salmonella, mientras que las muestras de alimento y recepción de pollitos de un día de edad tuvieron los menores porcentajes de positividad. (Tabla 3 ).

Se identificaron 4 serotipos de Salmonella: $S$. Infantis $(74.13 \%) \quad S$ Liverpool $(6.89 \%)$ $S$ Amsterdam (1.72\%) y $S$ Uganda (1.72\%). Dos cepas de Salmonella no pudieron ser

Tabla 2. Lista de Antimicrobianos utilizados y estándares de interpretación

\begin{tabular}{|c|c|c|c|c|c|}
\hline \multirow[b]{2}{*}{ Antimicrobiano } & \multirow[b]{2}{*}{ Abreviatura } & \multirow[b]{2}{*}{ Concentración } & \multicolumn{3}{|c|}{ Estándar Interpretativo (mm) } \\
\hline & & & $\mathbf{R}$ & $\mathbf{I}$ & $\mathbf{S}$ \\
\hline \multicolumn{6}{|l|}{ Aminoglucósidos } \\
\hline Gentamicina & G & 10 & 12 & $13-14$ & 15 \\
\hline Estreptomicina & $\mathrm{E}$ & 10 & 11 & $12-14$ & 15 \\
\hline \multicolumn{6}{|l|}{ B-lactamicos } \\
\hline Penicilina & $\mathrm{P}$ & 10 & 14 & - & 15 \\
\hline Ampicilina & M & 10 & 13 & $14-16$ & 17 \\
\hline Amoxicilina-Ác. Clavulánico & A & 20 & 13 & $14-17$ & 18 \\
\hline \multicolumn{6}{|l|}{ Cefalosporina 1era generación } \\
\hline Cefalotina & $\mathrm{F}$ & 30 & 14 & $15-17$ & 18 \\
\hline \multicolumn{6}{|l|}{ Cefalosporina 3era generación } \\
\hline Cefotaxima & $\mathrm{X}$ & 30 & 22 & $23-25$ & 26 \\
\hline \multicolumn{6}{|l|}{ Macrólido } \\
\hline Azitromicina & $\mathrm{Z}$ & 15 & 12 & - & 13 \\
\hline \multicolumn{6}{|l|}{ Fluoroquinolona } \\
\hline Ciprofloxacina & $\mathrm{C}$ & 5 & 20 & $21-30$ & 31 \\
\hline \multicolumn{6}{|l|}{ Tetraciclina } \\
\hline Tetraciclina & $\mathrm{T}$ & 30 & 11 & $12-14$ & 15 \\
\hline \multicolumn{6}{|l|}{ Nitrofurano } \\
\hline Nitrofurantoína & $\mathrm{N}$ & 300 & 14 & $15-16$ & 17 \\
\hline \multicolumn{6}{|l|}{ Fenicol } \\
\hline Cloranfenicol & $\mathrm{L}$ & 30 & 12 & $13-17$ & 18 \\
\hline \multicolumn{6}{|l|}{ Trimetopin-sulfametoxazol } \\
\hline Trimetopin-sulfametoxazol & S & 25 & 10 & $11-15$ & 16 \\
\hline
\end{tabular}


REVISTA ECUATORIANA DE MEDICINA Y CIENCIAS BIOLOGICAS

Tabla 3. Muestras positivas al aislamiento de Salmonella

\begin{tabular}{llcl}
\hline Sitio de muestreo & Tipo de muestra & Número muestras & $\begin{array}{l}\text { Número de muestras } \\
\text { positivas (\%) }\end{array}$ \\
\hline Planta de Alimento & Alimento & 194 & $8(4.1)$ \\
Crianza en granja & Recepción & 29 & $1(3.4)$ \\
& Hisopos de Barrido & 12 & $8(66.7)$ \\
& Heces & 6 & $3(50.0)$ \\
Planta de Faenamiento & Piel antes de pre enfriamiento & 24 & $19(79.2)$ \\
& Piel después de enfriamiento & 24 & $19(79.2)$ \\
\hline Total & & 289 & $58(20.1)$ \\
\hline
\end{tabular}

serotipificadas con los antisueros disponibles. Todas las cepas de Salmonella de muestras de piel tomadas antes del tanque de pre enfriamiento y después del tanque de enfriamiento pertenecieron al serotipo Infantis (Tabla 4).

Susceptibilidad antimicrobiana de Salmonella.La mayoría de las cepas de Salmonella presentaron fenotipos multiresistentes con resistencia a por lo menos dos antibióticos, con excepción de $S$. Uganda que no presentó resistencia a ningún antibiótico. Se identificaron 15 patrones de resistenciasiendo el grupo 4 el patrón de resistencia más frecuente. Las cepas de $S$. Infantis presentaron los patrones de resistencia con el mayor número de antibióticos (Tabla 5).
Las cepas de Salmonella de muestras de piel tomadas antes del tanque de pre enfriamiento y después del tanque de enfriamiento mostraron los mayores porcentajes de resistencia con patrones de 12 antibióticos, seguido de las muestras de hisopos de barrido. En tanto que las muestras de alimento presentaron los patrones de menor resistencia con 3 antibióticos. Una muestra de heces fue la única resistente a estreptomicina (Tabla 6).

\section{DISCUSIÓN}

Salmonella se encontró en todas las etapas de la empresa avícola. Los datos muestran que en la fábrica de alimento balanceado la bacteria estuvo presente en el $4.1 \%(n=194)$ de muestras de piensos e ingredientes. Estos resultados

Tabla 4. Serotipos de Salmonella

\begin{tabular}{lccc}
\hline Tipo de Muestra & $\begin{array}{c}\text { Aislamientos } \\
\text { Salmonella }\end{array}$ & Serotipo (n) & $\%$ \\
\hline Alimento & & Infantis (2) & 25 \\
& 8 & Liverpool (4) & 50 \\
& & Ámsterdam (1) & 12,5 \\
Recepción & 1 & Salmonella* (1) & 12,5 \\
Hisopos de barrido & 8 & Salmonella** (1) & 100 \\
& & Infantis (7) & 87,5 \\
Heces & 3 & Uganda (1) & 12,5 \\
Piel antes de pre enfriamiento & 19 & Infantis (3) & 100 \\
Piel después de enfriamiento & 19 & Infantis (19) & 100 \\
\hline
\end{tabular}

*Aglutinación antisuero Poly A, Antisuero somático O: 1,9,12.

**Aglutinación con antisuero Poly C. 
Tabla 5. Serotipos y perfil de resistencia

\begin{tabular}{|c|c|c|c|c|c|}
\hline \multirow[b]{2}{*}{ Patrón } & \multirow[b]{2}{*}{ Perfil de resistencia } & \multirow[b]{2}{*}{ Serotipo } & \multicolumn{2}{|c|}{ Aislamientos } & \multirow[b]{2}{*}{ Origen de la muestra (n) } \\
\hline & & & $\mathbf{n}$ & $(\%)$ & \\
\hline$\overline{1}$ & GPMAFLXCSTN & $S$. Infantis & 6 & 10,5 & $\mathrm{Hb}(1) ; \mathrm{Pa}(1) ; \mathrm{Pd}(4)$ \\
\hline 2 & PMFLXZCSTN & $S$. Infantis & 2 & 3,5 & $\mathrm{~Pa}(1) ; \mathrm{Pd}(1)$ \\
\hline 3 & GPMFLXCSTN & $S$. Infantis & 1 & 1,8 & $\mathrm{~Pa}(1)$ \\
\hline 4 & PMFLXCSTN & $S$. Infantis & 26 & 45,6 & $\mathrm{Hb}(4) ; \mathrm{H}(1) ; \mathrm{Pa}(11) ; \mathrm{Pd}(10)$ \\
\hline 5 & EPMFLXCTN & $S$. Infantis & 1 & 1,8 & $\mathrm{H}(1)$ \\
\hline 6 & GPMFLXSTN & $S$. Infantis & 1 & 1,8 & $\mathrm{~Pa}(1)$ \\
\hline 7 & PMFLXSTN & $S$. Infantis & 5 & 8,8 & $\mathrm{Hb}(2) ; \mathrm{Pa}(2) ; \mathrm{Pd}(1)$ \\
\hline 8 & PMFXCSTN & $S$. Infantis & 2 & 3,5 & $\mathrm{H}(1) ; \mathrm{Pa}(1)$ \\
\hline 9 & PMAFXZCN & Salmonella spp. & 1 & 1,8 & $\mathrm{R}(1)$ \\
\hline 10 & GLCSTN & $S$. Infantis & 3 & 5,3 & $\mathrm{~Pa}(1) ; \mathrm{Pd}(2)$ \\
\hline 11 & LXCSTN & $S$. Infantis & 1 & 1,8 & $\mathrm{~Pa}(1)$ \\
\hline 12 & $\mathrm{ZCN}$ & $S$. Infantis, $S$. Amsterdam & 2 & 3,5 & $\mathrm{Al}(2)$ \\
\hline 13 & $\mathrm{ZN}$ & Salmonella spp. & 1 & 1,8 & $\mathrm{Al}(1)$ \\
\hline 14 & $\mathrm{~N}$ & $S$. Liverpool & 3 & 5,3 & $\mathrm{Al}(3)$ \\
\hline 15 & $\mathrm{C}$ & $S$. Infantis, $S$. Liverpool & 2 & 3,5 & $\mathrm{Al}(2)$ \\
\hline Total & & & 57 & 100 & \\
\hline
\end{tabular}

contrastan con los niveles de contaminación por Salmonella obtenidos en Costa Rica de 5.4\% $(\mathrm{n}=1420)$ y en Tanzania con $29.4 \% \quad(n=197)$ de positividad (Mdemua et al. 2016, Molina et al. 2016). Por el contrario, los resultados reportados son miliares a los encontrados en Brasil y España en donde la bacteria ha sido aislada en el $4.9 \%(n=1269)$ y $4.8 \%(n=3844)$ de las muestras analizadas, respectivamente (Torres et al., 2011, Pellegrini et al. 2015).

Por otra parte, el patógeno se aisló únicamente de materias primas de origen animal $(6.7 \%)$, siendo la harina de carne $(22.2 \%)$ el ingrediente más contaminado. Estos datos difieren de los reportados en Turquía, en donde el 3.5\% $(\mathrm{n}=400)$ de harinas de origen animal presentaron contaminación por Salmonella (Kutay et al. 2016). Sin embargo, en China se reportó $35.7 \%(n=14)$ de contaminación en harina de carne (Yang et al. 2016). La presencia de la bacteria en este tipo de ingredientes puede deberse a la utilización de subproductos animales infectados que han sido sometidos a un proceso de rendering ineficaz (Wierup 2013).

Hay que tomar en cuenta que la comparación de datos acerca de la prevalencia de Salmonella en materias primas y/o alimento terminado resultan difíciles de realizar, debido a las diferencias en los métodos de muestreo y de diagnóstico aplicados en cada investigación (EFSA 2008). En granja, el microorganismo estuvo presente en el $3.5 \%(\mathrm{n}=29)$ de muestras de papel de recepción,
$66.7 \%(n=12)$ en hisopados de barrido y $50 \%(n=6)$ en muestras de ciego. La presencia de Salmonella a nivel de granja es variable. Así, en Brasil, el microrganismo se aisló del 3,6\% (n=28) de muestras de forros de cajas de transporte de pollos y en España el $31.2 \%(n=64)$ de muestras de meconio y forros de papel fueron positivas a Salmonella (Marin, et al. 2011). En contraste, Adesiyun et al. (2014) obtuvieron un resultado negativo en todas las muestras de meconio $(n=20)$. La presencia de Salmonella en pollitos de un día de edad sugeriría la posibilidad de una transmisión vertical desde las reproductoras o una transmisión horizontal desde la incubadora o durante el transporte de los pollitos hacia las granjas.

Los resultados de la presencia de Salmonella en las heces de pollos son similares a los reportados en Colombia en donde se detectó una prevalencia de $65 \%$ $(n=315)$ (Donado-Godoy et al. 2012). Sin embargo, en China este microorganismo fue aislado del 11.4\% $(\mathrm{n}=290)$ de muestras de ciegos y en España la prevalencia del patógeno en granjas de pollos de engorde fue del $1.02 \%$ $(n=6577)$ (Cui et al. 2016, Lamas et al. 2016). En Estados Unidos Salmonella se detectó en el $43.1 \%$ $(\mathrm{n}=109)$ y $13.6 \%(\mathrm{n}=220)$ de muestras de hisopado de barrido y heces, respectivamente (Berghaus et al. 2013).

Adicionalmente, en Brasil esta bacteria estuvo presente en el $5.0 \%(\mathrm{n}=40)$ de muestras de hisopados 
REVISTA ECUATORIANA DE MEDICINA Y CIENCIAS BIOLOGICAS

Tabla 6. Resistencia antimicrobiana en cepas de Salmonella

\begin{tabular}{|c|c|c|c|c|c|c|c|}
\hline \multirow[b]{2}{*}{ Antimicrobiano } & \multicolumn{6}{|c|}{ Aislados con fenotipos resistentes en cada punto de muestreo (\%) } & \multirow[b]{2}{*}{ Total $(\%)$} \\
\hline & Alimento & Recepción & $\begin{array}{l}\text { Hisopos de } \\
\text { barrido }\end{array}$ & Heces & $\begin{array}{l}\text { Piel antes pre } \\
\text { enfriamiento }\end{array}$ & $\begin{array}{l}\text { Piel después } \\
\text { enfriamiento }\end{array}$ & \\
\hline \multicolumn{8}{|l|}{ Aminoglucósidos } \\
\hline Gentamicina & 0 & 0 & $1(12.5)$ & 0 & $3(15.8)$ & $7(36.8)$ & $11(18.9)$ \\
\hline Estreptomicina & 0 & 0 & 0 & $1(33.3)$ & 0 & 0 & $1(1.7)$ \\
\hline \multicolumn{8}{|l|}{ B-lactamicos } \\
\hline Penicilina & 0 & $1(100)$ & $7(87.5)$ & $3(100)$ & $17(89.5)$ & $17(89.5)$ & 45 (77.6) \\
\hline Ampicilina & 0 & $1(100)$ & $7(87.5)$ & $3(100)$ & $17(89.5)$ & $17(89.5)$ & $45(77.6)$ \\
\hline Amoxicilina-Ác, Clavulánico & 0 & $1(100)$ & $1(12.5)$ & 0 & $1(5.3)$ & $4(21.1)$ & $7(12.1)$ \\
\hline \multicolumn{8}{|l|}{ Cefalosporina 1era generación } \\
\hline Cefalotina & 0 & $1(100)$ & $7(87.5)$ & $3(100)$ & $17(89.5)$ & $17(89.5)$ & $45(77.6)$ \\
\hline \multicolumn{8}{|l|}{ Cefalosporina 3era generación } \\
\hline Cefotaxima & 0 & $1(100)$ & $7(87.5)$ & $3(100)$ & $18(94.7)$ & $17(89.5)$ & $46(79.3)$ \\
\hline \multicolumn{8}{|l|}{ Macrólido } \\
\hline Azitromicina & $3(37.5)$ & $1(100)$ & 0 & 0 & $1(5.3)$ & $1(5.3)$ & $6(10.3)$ \\
\hline \multicolumn{8}{|l|}{ Fluoroquinolona } \\
\hline Ciprofloxacina & $5(62.5)$ & $1(100)$ & $5(62.5)$ & $3(100)$ & $1(5.3)$ & $1(5.3)$ & $16(27.6)$ \\
\hline \multicolumn{8}{|l|}{ Tetraciclina } \\
\hline Tetraciclina & 0 & 0 & $7(87.5)$ & $3(100)$ & $19(100)$ & $19(100)$ & $48(82.8)$ \\
\hline \multicolumn{8}{|l|}{ Nitrofurano } \\
\hline Nitrofurantoína & $6(75)$ & $1(100)$ & $7(87.5)$ & $3(100)$ & $19(100)$ & $19(100)$ & $55(94.8)$ \\
\hline \multicolumn{8}{|l|}{ Fenicol } \\
\hline Cloranfenicol & 0 & 0 & $7(87.5)$ & $2(66.7)$ & $18(94.7)$ & $19(100)$ & $46(79.3)$ \\
\hline \multicolumn{8}{|l|}{ Trimetopin-sulfametoxazol } \\
\hline Trimetopin-sulfametoxazol & 0 & 0 & $7(87.5)$ & $2(66.7)$ & $19(100)$ & $19(100)$ & $47(81)$ \\
\hline Total $(\%)^{* * *}$ & $3(23.1)$ & $8(61.5)$ & $11(84.6)$ & $10(76.9)$ & $12(92.3)$ & $12(92.3)$ & \\
\hline
\end{tabular}

de barrido colectados días antes de finalizarse la crianza y en Ecuador el 15.9\% $(n=388)$ de lotes de pollos fueron positivos a Salmonella (Giombelli y Abreu 2014, Vinueza-Burgos et al. 2016)

Las diferencias en la prevalencia de Salmonella reportada en el Ecuador puede deberse a que en el estudio realizado por Vinueza-Burgos et al. (2016) se abarcó un mayor número de empresas avícolas durante un año.

Además, las variaciones de la presencia de Salmonella en cada estudio se pueden atribuir a diferencias en las prácticas de crianza, terapia antibacteriana y/o condiciones de manejo y bioseguridad de las granjas.

En la planta de faenamiento no se encontraron variaciones en la cantidad de Salmonella aislada antes ni después del enfriamiento de las canales. Esto coincide Icon un estudio realizado en Costa Rica en donde el porcentaje de Salmonella en carcasas tomadas antes y después del enfriamiento fue el mismo (Rivera et al. 2014).

No obstante, en otras investigaciones el aislamiento de esta bacteria disminuye después de la inmersión de las carcasas en el tanque de enfriamiento. Así en Estados Unidos, se reportó una contaminación de $18.2 \%(n=330)$ antes y $2.4 \%(n=330)$ después del ingreso de las canales al tanque de enfriamiento (Berghaus et al. 2013). De igual manera, Cox et al. (2014) encontraron una contaminación por Salmonella del 52\% $(\mathrm{n}=40)$ antes del enfriamiento y $5 \%(n=40)$ después del enfriamiento de las carcasas en agua de inmersión.Adicionalmente, algunos países de Latinoamérica han reportado menores porcentajes de contaminación con Salmonella en carcasas al final del proceso de faenamiento. Así, en Brasil Salmonella se asiló en el 2.2\% $(\mathrm{n}=452)$ de carcasas colectadas después ser empaquetadas y en Venezuela el 23.8\% ( $\mathrm{n}=91)$ de canales antes de ser empacadas presentaron contaminación (Pérez et al. 2004, Brizio y Prentice 2015).

Son varios los factores que influyen en la contaminación de las canales; Rasschaert et al. (2007), mencionan que la presencia de este microorganismo en la línea de procesamiento de la planta de faenamiento puede influir en la contaminación cruzada de las carcasas. Además, la presencia y cantidad de contenido fecal en las cubetas de transporte, también son un factor de riesgo para la posterior 
contaminación de las canales por Salmonella (Heyndrickx et al. 2002). Otro punto a considerarse es la utilización de cloro con el fin de reducir la cantidad de Salmonella presente en las carcasas. En general, los estudios muestran que el uso de cloro en el agua de inmersión puede disminuir los niveles del patógeno en las canales de pollo (Logue et al. 2003, Nagel et al. 2013). No obstante, la eficacia de los tratamientos a base de cloro puede reducirse debido a la gran cantidad de materia orgánica, $\mathrm{pH}$ alcalino o una insuficiente concentración de cloro en los tanques de enfriamiento (Lillard 1979, Loretz, et al. 2016).

El serovar Infantis fue el más frecuente y constante a lo largo de la línea de crianza (86.2\%). La prevalencia de los serotipos de Salmonella en aves de corral puede variar según el país y condiciones climáticas (Wallis y Barrow 2005). Es así que en la UE $S$. Infantis $(29.2 \%), S$. Enteritidis $(13.6 \%)$ y $S$. Kentucky $(6.2 \%)$ fueron los serotipos más frecuentes encontrados en carcasas de pollo (EFSA 2010). De igual manera, a nivel de granja $S$. Infantis (22\%) fue el serovar más común seguido por $S$. Mbandaka (14.6\%), S. Thompson $(10.6 \%)$ y $S$. Enteritidis (4.9\%) (EFSA 2015). En otros lugares del mundo como Irán $(1.1 \%$; $\mathrm{n}=150)$ y Serbia $(1.5 \% ; \mathrm{n}=550)$ todas las cepas de Salmonella aisladas de carcasas de pollo fueron identificadas como $S$. Infantis ( Zare Bidaki et al. 2013, Rašeta et al. 2014).

En contraste, en Bangladesh, $S$. Enteritidis y $S$. Typhimurium fueron las serovariedades más prevalentes aisladas a partir de muestras de fluido intestinal e hisopados cloacales (Akond et al. 2012), mientras que en el Estado de Kuwait, S. Enteritidis fue el serotipo más común en muestras de granja y planta de faenamiento (Al-Zenki et al. 2007).

Los serotipos Liverpool, Amsterdam y Uganda fueron aislados con menor frecuencia de muestras de alimento y granja. Estos resultados son similares al de otras investigaciones en las cuales $S$. Liverpool $(0.2 \%), S$. Amsterdam (3.6\%) y $S$. Uganda (7.3\%) fueron las serovariedades menos comunes encontradas a nivel de granja (van Asselt, et al. 2009).

La distribución de serotipos de Salmonella cambió al final de la cadena productiva, siendo $S$. Infantis la serovariedad predominante. Ello probablemente se deba a que la supervivencia de Salmonella enterica puede variar según el serotipo (Andino et al. 2014). Igualmente, la exposición repetida de la bacteria a un determinado ambiente hostil puede dar como re- sultado el surgimiento de cepas altamente resistentes a ese entorno (Humphrey 2004). Sin embargo, la realización de un muestreo a nivel nacional podría generar mayor información en cuanto a la diversidad y prevalencia de serovariedades de Salmonella presentes en carcasas de pollos de engorde.

La existencia de cepas de Salmonella resistentes a los antimicrobianos representa un grave problema de salud pública. Varios productos alimenticios pueden ser fuente de Salmonella, sin embargo, se considera que los casos de salmonelosis en humanos están estrechamente asociados al consumo de productos de origen aviar (FAO/WHO 2009, Antunes, et al .2016).

En el presente estudio las cepas aisladas de Salmonella de varios procesos de la industria avícola presentaron patrones multiresistentes que tuvieron de 2 a 11 antibióticos. Las cepas de Salmonella aisladas a partir de piensos y sus materias primas mostraron resistencia a 3 antibióticos: nitrofurantoína (75\%), ciprofloxacina $(62.5 \%)$ y azitromicina $(37.5 \%)$. Estos resultados difieren con los reportados en Estados Unidos (EU), donde la mayor parte de cepas de Salmonella aisladas en alimento aviar fueron resistentes a sulfisoxazol, gentamicina,estreptomicina, tetraciclina y trimetopin-sulfametoxazol (Sanad et al. 2015). En Colombia un estudio reporta que las cepas de Salmonella aisladas de muestras de alimento presentaron mayor resistencia a amikacina, cefalotina, cefoxitina, cefuroxima y gentamicina (Rodriguez et al. 2015).

Se debe considerar que la sola presencia de cepas de Salmonella resistentes a antibióticos en esta fase de la industria avícola puede tener un gran impacto en la contaminación y propagación de esta bacteria en las aves durante la cadena productiva con la subsecuente transmisión a alimentos de consumo humano.

Durante la crianza en granja de los pollos de engorde las cepas positivas a Salmonella mostraron patrones de resistencia con un mayor número de antibióticos que los encontrados en el alimento. En este grupo los antibióticos con mayores reportes de cepasresistentes fueron: penicilina $(91.7 \%)$, ampicilina $(91.7 \%)$, cefalotina (91.7\%), cefotaxima (91.7\%), nitrofurantoína $(91.7 \%)$, tetraciclina $(83.3 \%)$ y cloranfenicol (75\%). Estos datos son parcialmente similares a los reportados en países como Estados Unidos, Corea y Colombia, en donde estudios reali- 
zados con muestras ambientales de granjas avícolas demuestran que gran parte de las cepas de Salmonella mostraron mayor resistencia a ampicilina, tetraciclina, ácido nalidíxico, ceftioufur, cefalotina, cloranfenicol, nitrofurantoína, trimetopin-sulfametoxazol (Alali et al. 2010, Im et al. 2015, Rodriguez et al. 2015). Según la Autoridad Europea de Seguridad Alimentaria la adquisición de genes de resistencia de Salmonella probablemente haya ocurrido por conjugación con otras enterobacterias mediante la transferencia de plásmidos (EFSA 2014). La resistencia de cepas de Salmonella a $\beta$-lactámicos de amplio espectro ha sido ampliamente estudiada. En la actualidad se conoce que las betalactamasas involucradas están codificadas por varios grupos de genes, entre los cuales se puede mencionar a AmpC, bla ${ }_{\mathrm{CMY}}$ bla $_{\mathrm{TEM}}$ y bla $\mathrm{CTX}_{\mathrm{CT}}$ entre los más prevalentes (Choi et al. 2015, Fitch et al. 2015, Katoh et al. 2015).

La aparición de bacterias productoras de betalactamasas de espectro extendido en aves de corral ecuatoriana es de particular interés para la salud pública ya que estos antibióticos figuran en la lista de medicamentos esenciales para la OMS (WHO 2013) limitando las opciones para el tratamiento de la salmonelosis humana.

Las cepas de Salmonella provenientes de muestras de piel de pollo de engorde destinado a consumo humano presentaron los fenotipos con mayor resistencia a los antibióticos. Estas cepas tuvieron resistencia a todos los antibióticos utilizados, excepto a estreptomicina. Los antibióticos con mayor frecuencia de resistencia fueron tetracicilina, nitrofurantoína, trimetopin-sulfametoxazol, cloranfenicol, cefotaxima, cefalotina, ampicilina $\mathrm{y}$ penicilina. Múltiples investigaciones realizadas en otros países como en la UE y EU se reportan que las cepas de Salmonella aisladas a partir de carne de pollo presentan mayores índices de resistencia a tetraciclina, ampicilina, estreptomicina, ciprofloxacina, ácido nalidíxico, sulfonamidas, ceftiofur (FDA 2013, EFSA 2015). Países latinoamericanos como México, Colombia y Brasil reportan que las cepas de Salmonella aisladas de carne de pollo muestran resistencias más altas a ampicilina, tetraciclina, cloranfenicol, trimetoprim-sulfametoxazol, enrofloxacina, ceftiofur, entre otros (Miranda et al. 2009, Donado-Godoy et al. 2015, Mattiello et al. 2015).

La resistencia elevada a tetraciclina en este estudio puede atribuirse al uso frecuente de estos antimi- crobianos en la producción animal y se ha reportado con frecuencia en varios países (Parveen et al. 2007, Maka, Maćkiw, Ściezyńska, Pawłowska y Popowska 2014, Choi et al. 2015, Quesada, et al. 2016).

Los altos niveles de resistencia de Salmonella en carne de pollo mostrados en este estudio pueden representar un riesgo significativo para la salud pública en el Ecuador. Esto se ha reportado en varios estudios que demuestran la importancia de Salmonella de origen aviar en la epidemiología clínica de la salmonelosis humana (Medeiros et al. 2011, Katoh et al. 2015, Quesada et al. 2016, Voss-Rech et al. 2016).

Treinta y nueve muestras procedentes de la etapa de crianza y de faenamiento comparten cuatro patrones de resistencia antimicrobiana. La contaminación de Salmonella procedente de contenido gastrointestinal puede ocurrir durante las operaciones de sacrificio, ocasionando la contaminación de las carcasas, lo que podría justificar los patrones de resistencia en las muestras. Estudios similares han reportado un aumento en la contaminación de las carcasas después de la evisceración (Von Rückert, et al. 2009, Wang et al. 2013, Giombelli et al. 2015).

Por otro lado, las cepas de S. Infantis aisladas en muestras de alimento no presentaron los mismos perfiles de resistencia que aquellas aisladas en muestras de crianza y faenamiento. Esto podría indicar que ciertas cepas de Salmonella están vinculadas a procesos específicos en la producción de pollos parrilleros. La realización de estudios complementarios permitiría determinar la relación genética entre estos aislados con mayor precisión.

Los resultados obtenidos en esta investigación proporcionan datos importantes acerca de la presencia, serotipos y resistencia a los antibióticos de cepas de Salmonella en una empresa avícola integrada del Ecuador, poniendo en relieve la necesidad de mejorar las medidas de bioseguridad y establecer programas de control de Salmonella que aseguren y verifiquen la calidad de la carne de pollo. De igual manera, es necesaria la implementación de medidas por parte de organismos oficiales que controlen el uso de antibacterianos en animales de abasto para prevenir el surgimiento de bacterias resistentes a los mismos. También se deberían realizar estudios que abarquen más integraciones avícolas, y la carne de pollo al menudeo para tener una idea más clara del riesgo de contaminación alimentaria en Ecuador. 


\section{AGRADECIMIENTOS}

Los autores expresan su agradecimiento a la Empresa Avícola que abrió sus puertas y apoyó a la realización de esta investigación. También agradecemos al personal técnico del laboratorio de Bacteriología de la Facultad de Medicina Veterinaria y Zootecnia de la Universidad Central del Ecuador.

\section{REFERENCIAS BIBLIOGRÁFICAS}

Adesiyun A, Webb L, Musai L, Louison B, Joseph G, Stewart-Johnson A, Samlal S, Rodrigo S. 2014. Survey of Salmonella Contamination in Chicken Layer Farms in Three Caribbean Countries. Journal of Food Protection 77(9): 1471-1480.

Akond M, Shirin M, Alam S, Hassan S, Rahman M, Hoq M. 2012. Frequency of drug resistant Salmonella spp. isolated from poultry samples in Bangladesh. Stamford Journal of Microbiology 2(1): $15-19$.

Al-Zenki S, Al-Nasser A, Al-Safar A, Alomirah H, Al-Haddad A, Hendriksen R, Aarestrup F. 2007. Prevalence and antibiotic resistance of Salmonella isolated from a poultry farm and processing plant environment in the State of Kuwait. Foodborne Pathogens and Disease 4(3): 367-73.

Alali W, Gaydashov R, Petrova E, Panin A, Tugarinov O, Kulikovskii A, Mamleeva D, Walls I, Doyle M. 2010. Prevalence and Distribution of Salmonella in Organic and Conventional Broiler Poultry Farms. Foodborne Pathogens and Disease 7(11): 1363-1371.

Andino A, Pendleton S, Zhang N, Chen W, Critzer F, Hanning I. 2014. Survival of Salmonella enterica in poultry feed is strain dependent. Poultry Science 93(2): 441-7.

Antunes P, Mourão J, Campos J, Peixe L. 2016. Salmonellosis: the role of poultry meat. Clinical Microbiology and Infection 22(2): 110-121.

Berghaus RD, Thayer SG, Law BF, Mild RM, Hofacre CL, Singer RS. 2013. Enumeration of Salmonella and Campylobacter spp. in Environmental Farm Samples and Processing Plant Carcass Rinses from Commercial Broiler Chicken Flocks. Applied and Environmental Microbiology 79(13): 4106-4114.

Brizio AP y Prentice C. 2015. Chilled broiler carcasses: a study on the prevalence of Salmonella, Listeria and Campylobacter. International Food Research Journal 22(1): $55-58$.

Choi D, Chon JW, Kim HS, Kim DH, Lim JS, Yim JH, Seo KH. 2015. Incidence, Antimicrobial Resistance, and Molecular Characteristics of Nontyphoidal Salmonella Including Extended-Spectrum $\beta$-Lactamase Producers in Retail Chicken Meat. Journal of Food Protection 78(11): 1932-7.

Clep R . 2010. Prevalence of mobile serovars of Salmonella spp. isolated from breeding hens, laying hens and broiler chickens in 2010. Scientific Works. C Series. Veterinary Medicine 58(4): 70-79.

CLSI. 2015. Performance Standards for Antimicrobial Susceptibility Testing. Clinical and Laboratory Standards Institute, 950. 35(3): 44-50.

CONAVE. 2013. Estadísticas avícolas. Página de Internet: http://www.conave.org/upload/ informacion/Estadisticas avicolas.pdf. Consultada: 18-diciembre-2015.

Cox NA, Buhr RJ, Smith DP, Cason JA, Rigsby LL, Bourassa DV, Fedorka-Cray P, Cosby D. 2014. Sampling naturally contaminated broiler carcasses for Salmonella by three different methods. Journal of Food Protection 77(3): 493495.

Cui M, Xie M, Qu Z, Zhao S, Wang J, Wang Y, He T, Wang H, Zuo Z, Wu C. 2016. Prevalence and antimicrobial resistance of Salmonella isolated from an integrated broiler chicken supply chain in Qingdao, China. Food Control 62: 270-276.

Da Silva N, Hirotomi M, Junqueira V, Silveira N, Da Silva M y Gomes R. 2013. Microbiological Examination Methods of Food and 
Water: A Laboratory Manual. CRC Press/ Balkema. London, UK. 484 pp.

Donado-Godoy P, Clavijo V, León M, Tafur MA, Gonzales S, Hume M, Alali W, Walls I, Lo Fo W, Danilo MA, Doyle MP. 2012. Prevalence of Salmonella on Retail Broiler Chicken Meat Carcasses in Colombia. Journal of Food Protection 75(6): 1134-1138.

Donado-Godoy P, Byrne B, León M, Castellanos R, Vanegas C, Coral A, Arevalo A, Clavijo V, Vargas M, Romero J, Tafur M, Pérez E, Smith W. 2015. Prevalence, resistance patterns, and risk factors for antimicrobial resistance in bacteria from retail chicken meat in Colombia. Journal of Food Protection 78(4): 751-9.

EFSA. 2008. Microbiological Risk Assessment in Feedingstuffs for Food-Producing Animals. EFSA Journal 720: 1-84.

EFSA. 2010. Analysis of the Baseline Survey on the Prevalence of Campylobacter in Broiler Batches and of Campylobacter and Salmonella on Broiler Carcasses in the EU, 2008, Part A: Campylobacter and Salmonella Prevalence Estimates. EFSA Journal $8(03)$.

EFSA. 2015. Trends and Sources of Zoonoses ,Zoonotic Agents and Food-Borne. EFSA Journal 13(1): 1-162.

EFSA, ECDC. 2013. The European Union Summary Report on Antimicrobial Resistance in Zoonotic and Indicator Bacteria from Humans, Animals and Food in 2011. EFSA Journal 11(5).

EFSA y ECDC. 2015a. EU Summary Report on Antimicrobial Resistance in Zoonotic and Indicator Bacteria from Humans, Animals and Food in 2013. European Food Safety Authority Journal 13(2).

EFSA y ECDC. 2015b. The European Union Summary Report on Trends and Sources of Zoonoses, Zoonotic Agents and Food-Borne Outbreaks in 2013." European Food Safety Authority Journal 13(1).

Eurosurveillance editorial team. 2014. The European Union Summary Report on antimi- crobial resistance in zoonotic and indicator bacteria from humans, animals and food 2012 published. Euro Surveillance : Bulletin Européen Sur Les Maladies Transmissibles=European Communicable Disease Bulletin 19(12): 20748.

FAO/WHO. 2009. Salmonella and Campylobacter in chicken meat, meeting report. Microbiological Risk Assesmment Series 19. Italy. $56 \mathrm{pp}$.

Fitch FM, Carmo-Rodrigues M, Oliveira VG, Gaspari MV, Dos Santos A, de Freitas JB y Pignatari AC. 2015. $\beta$-Lactam Resistance Genes: Characterization, Epidemiology, and First Detection of blaCTX-M-1 and blaCTX-M-14 in Salmonella spp. Isolated from Poultry in Brazil-Brazil Ministry of Agriculture's Pathogen Reduction Program. Microbial Drug Resistance 22(2): 164-71.

FDA. 2013. National Antimicrobial Resistance Monitoring System - Enteric Bacteria (NARMS): 2012 Executive Report. Rockville, MD: U.S. Department of Health and Human Services, Food and Drug Administration.

Giombelli A y Abreu M. 2014. Prevalence of Salmonella and Campylobacter on Broiler Chickens from Farm to Slaughter and Efficiency of Methods To Remove Visible Fecal Contamination. Journal of Food Protection 77(11): 1851-1859.

Giombelli A, Hammerschmitt D, Cerutti MF, Chiarini E, Landgraf M, Franco BD y Destro M. 2015. High pressure spray with water shows similar efficiency to trimming in controlling microorganisms on poultry carcasses. Poultry Science 94(10): 2589-95.

Guastalli B, Batista D, Souza A, Guastalli E, Lopes P, Almeida A, Prette N, Barbosa F, Stipp D, Freitas O. 2016. Evaluation of Disinfectants Used in Pre-Chilling water Tanks of Poultry Processing Plants. Revista Brasileira de Ciência Avícola 18(2): 217-224.

Gyles CL. 2008. Antimicrobial resistance in selected bacteria from poultry. Animal Health Research Reviews 9(2): 149-158. 
Herren RV. 2012. The Poultry Industry. In: The Science of Animal Agriculture: 76-95. New York.

Heyndrickx M, Vandekerchove D, Herman L, Rollier I, Grijspeerdt K, De Zutter, L. 2002. Routes for Salmonella contamination of poultry meat: epidemiological study from hatchery to slaughterhouse. Epidemiology and Infection 129(2): 253-265.

Humphrey T. 2004. Science and society: Salmonella, stress responses and food safety. Nature Reviews Microbiology 2(6): 504-509.

Im MC, Jeong SJ, Kwon YK, Jeong OM, Kang MS, Lee YJ. 2015. Prevalence and characteristics of Salmonella spp. isolated from commercial layer farms in Korea. Poultry Science 94(7): 1691-8.

Katoh R, Matsushita S, Shimojima Y, Ishitsuka R, Sadamasu K , Kai A. 2015. Serovars and Drug-Resistance of Salmonella Strains Isolated from Domestic Chicken Meat in Tokyo (1992-2012). The Journal of the Japanese Association for Infectious Diseases 89(1): 46-52.

Kutay HC, Dümen E, Keser O, Bilgin AŞ, Ergin S y Kocabağli N. 2016. Prevalence and Antimicrobial Susceptibility of Salmonella in Rendered Animal Products Used in Poultry Feed in Turkey. Kafkas Universitesi Veteriner Fakultesi Dergisi 22(6): 909-916.

Lamas A, Fernandez-No I, Miranda JM, Vázquez B, Cepeda A, Franco CM. 2016. Prevalence, molecular characterization and antimicrobial resistance of Salmonella serovars isolated from northwestern Spanish broiler flocks (2011-2015). Poultry Science 95(9): 2097-2105.

Lee SK., Choi D, Kim HS, Kim DH, Seo KH. 2016. Prevalence, Seasonal Occurrence, and Antimicrobial Resistance of Salmonella spp. Isolates Recovered from Chicken Carcasses Sampled at Major Poultry Processing Plants of South Korea. Foodborne Pathogens and Disease 13(10): 544-550.

Lillard HS. 1979. Levels of chlorine and chlorine dioxide of equivalent bactericidal effect in poultry processing water. Journal of Food Science 44(6): 1594-1597.

Logue CM, Sherwood JS, Olah PA, Elijah LM, Dockter MR. 2003. The incidence of antimicrobial-resistant Salmonella spp. on freshly processed poultry from US Midwestern processing plants. Journal of Applied Microbiology 94(1): 16-24.

Loretz M, Stephan R, Zweifel C. 2010. Antimicrobial activity of decontamination treatments for poultry carcasses: A literature survey. Food Control 21(6): 791-804.

Mainali C, McFall M, King R, Irwin R. 2014. Evaluation of Antimicrobial Resistance Profiles of Salmonella Isolates from Broiler Chickens at Slaughter in Alberta, Canada. Journal of Food Protection 77(3): 485-492.

Maka L, Maćkiw E, Ściezyńska H, Pawłowska K, Popowska M. 2014. Antimicrobial susceptibility of Salmonella strains isolated from retail meat products in Poland between 2008 and 2012. Food Control 36(1).

Marin C, Balasch S, Vega S, Lainez M. 2011. Sources of Salmonella contamination during broiler production in Eastern Spain. Preventive Veterinary Medicine 98(1): 39-45.

Mattiello SP, Drescher G, Barth VC, Ferreira CA, Oliveira SD. 2015. Characterization of antimicrobial resistance in Salmonella enterica strains isolated from Brazilian poultry production. Antonie van Leeuwenhoek 108(5): 1227-1238.

Mdemua S, Maina J, Ephraim Z. 2016. Isolation of Salmonella in Commercial Chicken Feeds in Ilala District. American Scientific Research Journal for Engineering, Technology, and Sciences 19(1): 1-8.

Medeiros M A, Oliveira D C, Rodrigues D, Freitas D R. 2011. Prevalence and antimicrobial resistance of Salmonella in chicken carcasses at retail in 15 Brazilian cities. Pan American Journal of Public Health 30(6): 555-60.

Miranda J M, Mondragón A C, Martinez B, Guarddon M, Rodriguez J A. 2009. Prevalen- 
ce and antimicrobial resistance patterns of Salmonella from different raw foods in Mexico. Journal of Food Protection 72(5): 966-971.

Molina A, Granados-Chinchilla F, Jiménez M, Acuña-Calvo M, Alfaro M, Chavarría G. 2016. Vigilance for Salmonella in Feedstuffs Available in Costa Rica: Prevalence, Serotyping and Tetracycline Resistance of Isolates Obtained from 2009 to 2014. Foodborne Pathogens and Disease 13(3): 119-127.

Moraes D, Andrade M, Duarte S, Bastos T, Arnhold E, Jayme V, Nunes I. 2016. Phenotypic and molecular detection of Salmonella sp. on growing, rearing and production phases in a commercial group of laying hens. Pesquisa Veterinária Brasileira 36(6): 503-508.

Nagel GM, Bauermeister LJ, Bratcher CL, Singh M, McKee SR. 2013. Salmonella and Campylobacter reduction and quality characteristics of poultry carcasses treated with various antimicrobials in a post-chill immersion tank. International Journal of Food Microbiology 165(3): 281-286.

OIE. 2012. OIE Manual of Diagnostic Tests and Vaccines for Terrestrial Animals. 7th edition. Francia.

OMS. 2014. Antimicrobial Resistance: Global Report on Surveillance. Página de Internet: http://www.who.int/drugresistance/documents/surveillancereport/en/. Consultada: 20-diciembre-2015

Parveen S, Taabodi M, Schwarz J G, Oscar T P, Harter-Dennis J, White D G. 2007. Prevalence and antimicrobial resistance of Salmonella recovered from processed poultry. Journal of Food Protection 70(11): 2466-2472.

Pellegrini D, Paim D, Lima G, Pissetti C, Kich J, Cardoso M. 2015. Distribution of Salmonella clonal groups in four Brazilian feed mills. Food Control 47: 672-678.

Pérez C, Rivera S, Pirela de Vera A, Rincón H, Mavárez Y, Román R. 2004. Aislamiento de Salmonella en canales de aves y evaluación de la efectividad de diferentes medios de enriquecimiento y selectivos. Revista Científica 14(2): 177-185.

Quesada A, Reginatto GA, Español AR, Colantonio LD, Burrone MS. 2016. Resistencia antimicrobiana de Salmonella spp aislada de alimentos de origen animal para consumo humano. Revista Peruana de Medicina Experimental Y Salud Publica 33(1): 32-44.

Rahmani M, Peighambari SM, Svendsen CA, Cavaco LM, Agersø Y, Hendriksen R S. 2013. Molecular clonality and antimicrobial resistance in Salmonella enterica serovars Enteritidis and Infantis from broilers in three Northern regions of Iran. BMC Veterinary Research 9(1): 66.

Rašeta $M$, Teodorović $\mathrm{V}$, Bunčić $\mathrm{O}$, Katić V, Branković Lazić I, Polaček V, Vidanović D. 2014. Antibiotic Resistance and Molecular Studies on Salmonella Enterica Subspecies Enterica

Serovar Infantis Isolated in Human Cases and Broiler Carcasses. Acta Veterinaria-Beograd 64(2) 257-268.

Rasschaert G, Houf K, De Zutter L. 2007. Impact of the Slaughter Line Contamination on the Presence of Salmonella on Broiler Carcasses. Journal of Applied Microbiology 103(2): 333-41.

Rivera W, Barquero E, Zamora R. 2014. Salmonella Contamination Risk Points in Broiler Carcasses during Slaughter Line Processing. Journal of Food Protection 77(12): 2031-34.

Rodriguez R, Fandiño C, Donado P, Guzmán L, Verjan N. 2015. Characterization of Salmonella from Commercial Egg-Laying Hen Farms in a Central Region of Colombia. Avian Diseases 59(1): 57-63.

Sanad YM, Johnson K, Park SH, Han J, Deck J, Foley SL, Kenney B, Ricke S, Nayak R. 2015. Molecular Characterization of Salmonella enterica serovars isolated from a turkey production facility in the absence of selective antimicrobial pressure. Foodborne Pathogens and Disease 13(2):80-7. 
Sodagari HR, Mashak Z, Ghadimianazar A. 2015. Prevalence and antimicrobial resistance of Salmonella serotypes isolated from retail chicken meat and giblets in Iran. The Journal of Infection in Developing Countries 9(5): 463-469.

Su LH., Chiu CH, Chu C, Ou JT. 2004. Antimicrobial Resistance in Nontyphoid Salmonella Serotypes: A Global Challenge. Clinical Infectious Diseases 39(4): 546-551.

Tîrziu E, Lazăr R, Sala C, Nichita I, Morar A, Şereș M, Imre K. 2015. Salmonella in Raw Chicken Meat from the Romanian Seaside: Frequency of Isolation and Antibiotic Resistance. Journal of Food Protection 78(5): 1003-1006.

Torres GJ, Piquer FJ, Algarra L, de Frutos C, Sobrino OJ. 2011. The prevalence of Salmonella enterica in Spanish feed mills and potential feed-related risk factors for contamination. Preventive Veterinary Medicine 98: 81-87.

van Asselt ED, Thissen JT, van der Fels-Klerx HJ. 2009. Salmonella serotype distribution in the Dutch broiler supply chain. Poultry Science 88(12): 2695-2701.

Vinueza C, Cevallos M, Ron L, Bertrand S, De Zutter L. 2016. Prevalence and Diversity of Salmonella Serotypes in Ecuadorian Broilers at Slaughter Age. PLoS ONE 11(7): e0159567.

Von Rückert D, Pinto P, Santos BM, Moreira M, Rodrigues AC. 2009. Pontos críticos de controle de Salmonella spp. no abate de frangos. Arquivo Brasileiro de Medicina Veterinaria E Zootecnia 61(2): 326-330.

Voss-Rech D, Vaz CS, Alves L, Coldebella A, Leão JA, Rodrigues DP, Back A. 2015. A temporal study of Salmonella enterica serotypes from broiler farms in Brazil. Poultry Science 94(3): 433-441.

Voss-Rech D, Potter L, Vaz CS, Pereira D, Sangioni L, Vargas Á, Botton S. 2016. Antimicrobial Resistance in Nontyphoidal Salmonella Isolated from Human and Poultry-Related Samples in Brazil: 20-Year Meta-Analysis. Foodborne Pathogens and Disease 20.
Wallis TS, Barrow PA. 2005. Salmonella Epidemiology and Pathogenesis in Food-Producing Animals. EcoSal Plus 1(2): 1-31.

Wang H, Ye K, Wei X, Cao J, Xu X, Zhou G. 2013. Occurrence, antimicrobial resistance and biofilm formation of Salmonella isolates from a chicken slaughter plant in China. Food Control 33(2): 378-384.

Wierup M. 2013. Salmonella in Feed. En: Salmonella in Domestics Animals, eds. Barrow P A y Methner U. London: C CAB International, 377-398.

World Health Organization. 2013. WHO Model List of Essential Medicines - 18th List (April 2013). Página de Internet: http:// www.who.int/medicines/publications/essentialmedicines/en/. Consultada: 21-febrero-2016.

World Health Organization. 2015. WHO estimates of the global burden of foodborne diseases: foodborne disease burden epidemiology reference group 2007-2015. Página de Internet: http://www.who.int/foodsafety/publications/foodborne disease/fergreport/ en/. Consultada: 20-enero-2017.

Yang S, Wu Z, Lin W, Xu L, Cheng L, Zhou L. 2016. Investigations into Salmonella contamination in feed production chain in Karst rural areas of China. Environmental Science and Pollution Research 24(2): 1372-1379.

Zare Bidaki M, Tehrani Pour A, Dadpour S, Gholizadeh, H. 2013. Prevalence of Salmonella in poultry carcasses serotypes in Birjand industrial slaughterhouses. Journal of Birjand University of Medical Sciences 20(2): 191-197. 